Working Paper 13-27

Statistics and Econometrics Series 23

July 2013
Departamento de Estadística

Universidad Carlos III de Madrid

Calle Madrid, 126

28903 Getafe (Spain)

Fax (34) 91 624-98-49

\title{
Data cloning estimation of GARCH and COGARCH models
}

\author{
J.Miguel Marín ${ }^{1}$ \\ María Teresa Rodríguez-Bernal ${ }^{2}$ \\ Eva Romero ${ }^{3}$
}

\begin{abstract}
GARCH models include most of the stylized facts of financial time series and they have been largely used to analyze discrete financial time series. In the last years, continuous time models based on discrete GARCH models have been also proposed to deal with non-equally spaced observations, as COGARCH model based on Lévy processes.

In this paper, we propose to use the data cloning methodology in order to obtain estimators of GARCH and COGARCH model parameters. Data cloning methodology uses a Bayesian approach to obtain approximate maximum likelihood estimators avoiding numerically maximization of the pseudo-likelihood function. After a simulation study for both GARCH and COGARCH models using data cloning, we apply this technique to model the behavior of some NASDAQ time series.
\end{abstract}

Keywords: GARCH, Continuous-time GARCH process, Lévy process, COGARCH, data cloning, Bayesian inference, MCMC algorithm.

1 Departamento de Estadística, Universidad Carlos III de Madrid, e-mail: jmmarin@est-econ.uc3m.es.

2 Departamento de Estadística e I.O., Universidad Complutense de Madrid, e-mail: mayter@mat.ucm.es

3 Departamento de Economía y Relaciones Internacionales, Universidad Europea de Madrid, e-mail: eva.romero@uem.es 


\title{
Data cloning estimation of GARCH and COGARCH models
}

\author{
Marín, J.M. ${ }^{1}$, Rodríguez-Bernal ${ }^{2}$, M.T. and Romero, E. ${ }^{3}$ \\ (1) Dep. de Estadística, U. Carlos III de Madrid, Spain \\ (2) Dep. de Estadística e Investigación Operativa, U. Complutense de Madrid, Spain. \\ (3) Dep. de Economía y Relaciones Internacionales, U. Europea de Madrid, Spain
}

\begin{abstract}
GARCH models include most of the stylized facts of financial time series and they have been largely used to analyze discrete financial time series. In the last years, continuous time models based on discrete GARCH models have been also proposed to deal with non-equally spaced observations, as COGARCH model based on Lévy processes.

In this paper, we propose to use the data cloning methodology in order to obtain estimators of GARCH and COGARCH model parameters. Data cloning methodology uses a Bayesian approach to obtain approximate maximum likelihood estimators avoiding numerically maximization of the pseudo-likelihood function. After a simulation study for both GARCH and COGARCH models using data cloning, we apply this technique to model the behavior of some NASDAQ time series.
\end{abstract}

Keywords: GARCH model, Continuous-time GARCH process, Lévy process, COGARCH model, data cloning, Bayesian inference, MCMC algorithm.

\section{Introduction}

Financial time series present usually stylized facts, namely, statistical irregularities. Mandelbrot (1963) pointed out that they usually show non-stationarity and variations that are similar to white noise. They also present heavy-tailed distributions with leverage and calendar effects which affect significantly the returns. These characteristics make useless the classical Box-Jenkins methodology.

Engle (1982) introduced ARCH models to include no constant variance. Later on, Bollerslev (1986) introduced GARCH models which generalize them by including an auto-regressive term to model the process variance. One of the most used methodology in financial time series are GARCH models applied in equally spaced data. However, long periods of time present holidays and weekends that may affect the behavior of series, therefore it is convenient to introduce models with unequally spaced or continuous time periods.

In this sense, Nelson (1990) defined a continuous GARCH model and derived a limiting GARCH $(1,1)$ model by adding GARCH innovations up. The resulting model was driven by two different and independent Brownian motions, one as the driving process and the other one, present in the variance process. However, Wang (2002) proved that the diffusion limit of Nelson was not asymptotically equivalent to a GARCH process, considering the Le Cam's deficiency distance.

Corradi (2000) modified Nelson's approximation in order to obtain an asymptotically equivalent discrete time GARCH process. In this case, both processes were driven by the same Brownian motion, but the limiting process was not a random process. Then, Kallsen and Taqqu (1998) introduced a continuous-time GARCH model where the processes are both driven by the same Brownian motion, and when it is applied in discrete periods of time it is equivalent to a standard GARCH model. This model is arbitrage free and complete, the volatility is constant over each time interval $[t, t+1]$ and they used this approach to determine pricing formulas for European options.

Kazmerchuk et al. (2005) derived a continuous time GARCH process driven also by one Brownian motion. In this case they defined the volatility process as a combination of volatility and price processes using a stochastic delay differential equation. Later on, Lorenz (2006) expanded the previous work obtaining some solutions for the model.

In this work, we will use the COGARCH model introduced by Klüppelberg et al. (2004). This model is based in a single Lévy process which drives both processes, and can also model jumps 
of the process. As Bollerslev (2008) pointed out, it is important that jumps could be valuable, because they exhibit a source of risk which is non-diversifiable.

In relation with the estimation techniques that can be used in this model, the method of moments was studied in Haug et al. (2007), who showed that the resulting estimators are consistent and asymptotically normal. On the other hand, estimators based in pseudo-maximum likelihood were studied by Maller et al. (2008), who approximated the COGARCH model with an embedded sequence of discrete-time GARCH series. Moreover, Müller (2010) used a Markov chain Monte Carlo (MCMC) based estimation procedure for the COGARCH model, driven by a compound Poisson process.

In this paper, we have applied data cloning methodology to estimate the parameters of the GARCH and COGARCH models. Data cloning methods have been developed to tackle with ecological complex models (see Lele et al. (2007) and Lele et al. (2010)). These methods give estimations of the parameters, by simulating the posterior distribution of them with a MCMC algorithm, which converge to those obtained by maximum likelihood $(M L)$ method. Using data cloning methodology it is not so relevant, in practice, which type of prior distribution is considered.

In section 2 we revise briefly the GARCH and COGARCH models that are used in this paper. In section 3 we present the main issues of the data cloning methodology as a general technique suitable for analyzing complex models, which permits to obtain frequentist estimators by means of a Bayesian framework. In Section 4 we introduce the data cloning estimators for the parameters of GARCH and COGARCH models and we use a simulation study for checking the accuray of the methodology. For this, we take simulated data from $\operatorname{GARCH}(1,1)$ and $\mathrm{COGARCH}$ models with fixed parameters, and we obtain estimators and confidence intervals from the data cloning application. Furthermore, we apply a Bayesian full procedure for those models with an aim of comparison with a pure Bayesian MCMC technique. As results seem to be reasonable, that permits to apply this methodology to real data and in section 5, we use the previous models in a real example of the NASDAQ stock index.

\section{GARCH and COGARCH Models}

In this section, we first revise some definitions and characteristics of GARCH models and the COGARCH model of Klüppelberg et al. (2004).

Stock returns have heavy-tailed distributions and, for that reason, the conditional variance is not constant. GARCH models are concerned with modelling volatility that fluctuates over time, which is very important as volatility is considered a measure of risk.

Definition 1. Assuming that volatilities $\sigma_{t}^{2}$ are random variables and $\epsilon_{t}$ is a sequence of random variables, independently and identically distributed, then $Y_{t}$ follow a model process $\operatorname{GARCH}(1,1)$ if

$$
Y_{t}=\sigma_{t} \epsilon_{t}
$$

where the volatility process is

$$
\sigma_{t}^{2}=\alpha_{0}+\alpha_{1} Y_{t-1}^{2}+\beta \sigma_{t-1}^{2}
$$

for $t \in \mathbb{N}, \alpha_{0}>0, \alpha_{1} \geq 0, \beta \geq 0$ and $\alpha_{1}+\beta>0$. We consider also that $\epsilon_{t}$ are normally distributed $N(0,1)$.

Definition 2. Let $\left(\Omega, \mathcal{F}, P,\left(\mathcal{F}_{t}\right)_{t \geq 0}\right)$ be a filtered probability space, where $\left(\mathcal{F}_{t}\right)_{t \geq 0}$ is the natural filtration of the background driving Lévy process $L=\left(L_{t}\right)_{t>0}$. Given an initial value $\sigma_{0}$, the COGARCH process $G=\left(G_{t}\right)_{t>0}$ and the variance process $\sigma^{2}=\left(\sigma_{t}^{2}\right)_{t>0}$ are defined by the stochastic differential equations

$$
\begin{aligned}
d G_{t} & =\sigma_{t} d L_{t} \\
d \sigma_{t+}^{2} & =\beta d t-\eta \sigma_{t}^{2} d t+\varphi \sigma_{t}^{2} d[L, L]_{t}
\end{aligned}
$$

where $t>0, G_{0}=0, \beta>0, \eta>0, \varphi \geq 0$ and $[L, L]_{t}$ is the quadratic variation of the Lévy process.

Maller et al. (2008) proved that this model can be expressed as a continuous time limit of a sequence of GARCH models. As the COGARCH model may be approximated by an appropriate 
set of GARCH processes, the parameters of the COGARCH model can be estimated using the relation between them.

In order to deal with the approximation to the COGARCH model, Maller et al. (2008) fixed an interval $[0, T]$, where $T>0$, and took the sequence of integer numbers $\left(N_{n}\right)_{n \geq 1}$ such that $\lim _{n \rightarrow \infty} N_{n}=\infty$ and a sequence of partitions

$$
0=t_{0}(n)<\ldots<t_{N_{n}}(n)=T
$$

which divide the interval $[0, T]$ into $N_{n}$ sub-intervals of length $\Delta t_{i}(n)=t_{i}(n)-t_{i-1}(n)$. It is assumed that $\Delta t(n)=\max _{i=1, \ldots, N_{n}} \Delta t_{i}(n) \longrightarrow 0$ when $n \rightarrow \infty$. Then, the corresponding discretetime processes $\left(G_{i, n}\right)_{i=1, \ldots, N_{n}}$ and $\left(\sigma_{i, n}^{2}\right)_{i=1, \ldots, N_{n}}$ are defined by

$$
\begin{aligned}
G_{i, n} & =G_{i-1, n}+\sigma_{i-1, n} \sqrt{\Delta t_{i}(n)} \epsilon_{i, n} \\
\sigma_{i, n}^{2} & =\beta \Delta t_{i}(n)+\left(1+\varphi \Delta t_{i}(n) \epsilon_{i, n}^{2}\right) e^{-\eta \Delta t_{i}(n)} \sigma_{i-1, n}^{2}, \quad i=1, \ldots, N_{n}
\end{aligned}
$$

where innovations $\epsilon_{i, n}$ are independent and identically distributed with $E\left(\epsilon_{i, n}\right)=0, V\left(\epsilon_{i, n}\right)=1$ and $\sigma_{0, n}^{2}=\sigma_{0}^{2}$.

The continuous-time versions of $G_{i, n}$ and $\sigma_{i, n}^{2}$ are defined as

$$
\begin{array}{lll}
G_{n}(t)=G_{i, n} & \text { in } \quad t \in\left[t_{i-1}(n), t_{i}(n)\right] \text { with } G_{n}(0)=0 \\
\sigma_{n}(t)^{2}=\sigma_{i, n}^{2} & \text { in } \quad t \in\left[t_{i-1}(n), t_{i}(n)\right]
\end{array}
$$

If $E\left(L_{1}\right)=0$ and $E\left(L_{1}^{2}\right)=1$, there exists for each $n \geq 1$ a sequence $\epsilon_{i, n}$ such that

$$
\rho\left(\left(G_{n}, \sigma_{n}^{2}\right),\left(G, \sigma^{2}\right)\right) \underset{n \rightarrow \infty}{\stackrel{P}{\longrightarrow}} 0
$$

where $\rho$ is the Skorokhod distance.

In this way, given a COGARCH model, there exists a sequence of GARCH models which converges to it. Then, the process $\left(G_{n}(t), \sigma_{n}(t)\right)$ can be considered as an approximation to the COGARCH model $(G(t), \sigma(t))$ for $n$ large enough.

Maller et al. (2008) used this approximation to fit the model to unequally spaced time data, by deriving a pseudo-maximum likelihood function and numerically maximizing it in order to estimate the corresponding parameters.

We take this pseudo-likelihood function to build the joint posterior distribution of the parameters, as a previous step to deal with the data cloning methodology. But, in this case, as the procedure is based on a MCMC algorithm, it is not necessary to numerically maximize the pseudolikelihood function as in Maller et al. (2008).

By other hand, we consider also full Bayesian procedures, based on MCMC techniques, for both GARCH and COGARCH cases regarding a comparison with the data cloning methodology.

Let us assume that data $G\left(t_{i}\right)$ are distributed as a COGARCH model, defined in (2) and (3), in time points $0=t_{0}<t_{1}<\ldots<t_{N}=T$.

Let $Y_{i}=G\left(t_{i}\right)-G\left(t_{i-1}\right)$ be returns and $\Delta t_{i}=t_{i}-t_{i-1}$ the time increments. As the process $\left(\sigma_{t_{i}}\right)_{i=1 \ldots, N}$ is Markovian, $Y_{i}$ is conditionally independent of the previous returns $Y_{i-1}, Y_{i-2}, \ldots$, given $\mathcal{F}_{t_{i-1}}$. The conditional expectation and variance of $Y_{i}$ given $\mathcal{F}_{t_{i-1}}$, by using Proposition 5.1 in Klüppelberg et al. (2004), are

$$
\begin{gathered}
E\left(Y_{i} \mid \mathcal{F}_{t_{i-1}}\right)=0 \\
\rho_{i}^{2}=\operatorname{Var}\left(Y_{i} \mid \mathcal{F}_{t_{i-1}}\right)=E\left(Y_{i}^{2} \mid \mathcal{F}_{t_{i-1}}\right)=\left(\sigma_{t_{i-1}}^{2}-\frac{\beta}{\eta-\varphi}\right)\left(\frac{e^{(\eta-\varphi) \Delta t_{i}}-1}{\eta-\varphi}\right)+\frac{\beta \Delta t_{i}}{\eta-\varphi}
\end{gathered}
$$

where $\left(L_{t}\right)_{t \geq 0}$ is a quadratic pure jump process with $E\left(L_{1}\right)=0, E\left(L_{1}^{2}\right)<\infty$ and $\left(\sigma_{t}^{2}\right)_{t \geq 0}$ is stationary.

To ensure the stationarity of the process it must be assumed that $E\left(L_{1}^{2}\right)=1$ and $E\left(\sigma_{0}^{2}\right)=\frac{\beta}{\eta-\varphi}$, with $\eta>\varphi$.

Then, assuming that $Y_{i}$ are conditionally $N\left(0, \rho_{i}^{2}\right)$, the pseudo-likelihood function for the observed returns $\mathbf{y}=\left(y_{1}, \ldots, y_{N}\right)$ is

$$
L(\beta, \varphi, \eta \mid \mathbf{y})=\left(\prod_{i=1}^{N} \frac{1}{\sqrt{2 \pi \rho_{i}^{2}}}\right) \exp \left\{-\sum_{i=1}^{N} \frac{y_{i}^{2}}{2 \rho_{i}^{2}}\right\}
$$


where

$$
\begin{aligned}
\sigma_{i}^{2} & =\beta \Delta t_{i}+e^{-\eta \Delta t_{i}} \sigma_{i-1}^{2}+\varphi e^{-\eta \Delta t_{i}}\left(\sqrt{\Delta t_{i}} \epsilon_{i, n} \sigma_{i-1}\right)^{2} \\
& =\beta \Delta t_{i}+e^{-\eta \Delta t_{i}} \sigma_{i-1}^{2}+\varphi e^{-\eta \Delta t_{i}} y_{i}^{2}
\end{aligned}
$$

The last equation is obtained by substituting $\sigma_{t_{i-1}}^{2}$ by $\sigma_{i-1}^{2}$ in (6) and assuming the discretization of the volatility process.

Maller et al. (2008) obtained the pseudo-maximum likelihood estimates by directly maximizing the pseudo-log-likelihood function for the initial value $\sigma_{0}^{2}=\frac{\beta}{\eta-\varphi}$.

\section{Data cloning methodology}

The data cloning method is a general technique to compute maximum likelihood estimates, along with their asymptotic variances, by means of a Bayesian MCMC procedure (see Lele et al. (2007) and Lele et al. (2010)).

In a MCMC procedure, once $\mathbf{y}=\left(y_{1}, \ldots, y_{N}\right)$ has been observed, any sample can be generated from the posterior distribution

$$
\pi(\beta, \varphi, \eta \mid \mathbf{y}) \propto L(\beta, \varphi, \eta \mid \mathbf{y}) \pi(\beta, \varphi, \eta)
$$

which is proportional to the product of the likelihood function $L(\beta, \varphi, \eta)$ and a given prior distribution $\pi(\beta, \varphi, \eta)$.

In data cloning methodology, samples are driven from the posterior distribution

$$
\pi^{(k)}(\beta, \varphi, \eta \mid \mathbf{y}) \propto[L(\beta, \varphi, \eta \mid \mathbf{y})]^{k} \pi(\beta, \varphi, \eta)
$$

which is proportional to the product of the $k$ th power of the likelihood function $[L(\beta, \varphi, \eta \mid \mathbf{y})]^{k}$ and the prior distribution $\pi(\beta, \varphi, \eta)$.

The expression $[L(\beta, \varphi, \eta \mid \mathbf{y})]^{k}$ is the likelihood for $k$ copies of the original data, and for $k$ large $\pi^{(k)}(\beta, \varphi, \eta \mid \mathbf{y})$ converges to a multivariate normal distribution with mean equal to the maximum likelihood $(M L)$ estimate of the parameters, and covariance matrix equal to $1 / k$ times the inverse of the Fisher information matrix for the $M L$ estimates (see Lele et al. (2007)).

In this way, after obtaining samples from the posterior distribution using a MCMC procedure, their sample means are computed which provide the maximum likelihood estimates of the parameters.

The data cloning algorithm can be summarized in the next steps

Step 1: Create $k$-cloned data set $\mathbf{y}^{(k)}=(\mathbf{y}, \mathbf{y}, \ldots, \mathbf{y})$, where the observed data vector is repeated $k$ times.

Step 2: Using an MCMC algorithm, generate random numbers from the posterior distribution that is based on a prior $\pi(\beta, \varphi, \eta)$ and the cloned data vector $\mathbf{y}^{(k)}=(\mathbf{y}, \mathbf{y}, \ldots, \mathbf{y})$, where the $k$ copies of $\mathbf{y}$ are assumed to be independent of each other. In practice, any proper prior distribution can be used.

Step 3: Compute the sample mean and variances of the values $(\theta)_{j}, j=1, \ldots, B$ (for $B$ iterations of the MCMC run) generated from the marginal posterior distribution. The $M L$ estimates of $(\theta)_{j}$ correspond to the posterior mean values and the approximate variances of the $M L$ estimates correspond to $k$ times the posterior variances.

\section{Data cloning estimation of GARCH and COGARCH mod- els}

In this section we apply the data cloning methodology to estimate the parameters of GARCH $(1,1)$ and COGARCH models. Usually, these models are studied either from a classical point of view, with moment estimators or with a pseudo-maximum likelihood procedure (see e.g. Francq and Zakoian (2010), Haug et al. (2007) and Maller et al. (2008)), or from a Bayesian point of view (see e.g. Ardia (2008) and Müller (2010)).

Interpretations of the estimators and credible intervals in the Bayesian approach are dependent of prior distributions. In this way, it is convenient to elicit accurate prior distributions. But in the data cloning approach any proper prior distribution can be used in pratice, and elicitation of prior distributions is not very relevant. 


\subsection{Data cloning estimation of $\operatorname{GARCH}(1,1)$ model}

We consider the estimation of the parameters of a $\operatorname{GARCH}(1,1)$ model under a data cloning methodology. The algorithm may be straightforwardly generalized in order to estimate the parameters of general GARCH models, by including the corresponding terms.

In the $\operatorname{GARCH}(1,1)$ model (1), the parameters of interest are $\alpha_{0}, \alpha_{1}$ and $\beta$. In order to guarantee the stationary of the process, the model must satisfy that $E\left[\ln \left(\alpha_{1} \eta_{t}^{2}+\beta\right)\right]<0$, and this permits to find boundaries over parameters.

Proposition 1. If a GARCH process satisfies that $E\left[\ln \left(\alpha_{1} \eta_{t}^{2}+\beta\right)\right]<0$, then the process is strictly stationary and $\alpha_{1}$ and $\beta$ are bounded between the following limits

$$
\begin{aligned}
\beta & \in(0,1) \\
\alpha_{1} & \in\left(0,2 e^{\gamma}\right)
\end{aligned}
$$

Proof. As $E\left[\ln \left(\alpha_{1} \eta_{t}^{2}+\beta\right)\right]<0$ and $\alpha_{1} \geq 0$

$$
E[\ln (\beta)] \leq E\left[\ln \left(\alpha_{1} \eta_{t}^{2}+\beta\right)\right]<0
$$

that is, $\beta \in(0,1)$, as in $(9)$.

In order to obtain (10), as $\beta \geq 0$

$$
E\left[\ln \left(\alpha_{1} \eta_{t}^{2}\right)\right] \leq E\left[\ln \left(\alpha_{1} \eta_{t}^{2}+\beta\right)\right]<0
$$

As $\eta_{t} \sim N(0,1)$, the characteristic function of $Y=\ln \left(\eta_{t}^{2}\right)$ is

$$
\begin{aligned}
\varphi_{Y}(t) & =E\left[e^{i t Y}\right]=E\left[e^{i t \ln \left(\eta_{t}^{2}\right)}\right]=E\left[\left(\eta_{t}^{2}\right)^{i t}\right] \\
& =\int_{0}^{\infty} \frac{1}{\sqrt{2 \pi}}\left(\eta_{t}^{2}\right)^{i t-1 / 2} e^{-\eta_{t}^{2} / 2} d \eta_{t}^{2} \\
& =\frac{1}{\sqrt{2 \pi}} \frac{\Gamma\left(\frac{2 i t+1}{2}\right)}{(1 / 2)^{\frac{2 i t+1}{2}}}
\end{aligned}
$$

which gives

$$
E\left[\ln \left(\eta_{t}^{2}\right)\right]=\frac{\varphi_{Y}^{\prime}(0)}{i}=\frac{\Gamma^{\prime}(1 / 2)}{\sqrt{\pi}}+\ln (2)
$$

and, as $\Gamma^{\prime}(t)=\Gamma(t) \psi(t)$, where $\psi(t)$ is the digamma function

$$
E\left[\ln \left(\eta_{t}^{2}\right)\right]=\psi(1 / 2)+\ln (2) \approx-\gamma-\ln (2)
$$

where $\gamma$ is the Euler-Mascheroni constant.

Substituting this value in (11), it is obtained that

$$
E\left[\ln \left(\alpha_{1} \eta_{t}^{2}\right)\right]=\ln \left(\alpha_{1}\right)+E\left[\ln \left(\eta_{t}^{2}\right)\right]=\ln \left(\alpha_{1}\right)-\gamma-\ln (2)<0
$$

and, hence, $\alpha_{1}<2 e^{\gamma}$.

In data cloning methodology, as the first step is to design a Bayesian MCMC procedure, we have to consider what the prior distributions of the parameters are; although the final results are not affected by the particular chosen prior distributions (see Lele et al. (2007)).

In this case, as vaguely informative distributions, we use the following uniform distributions taking into account the corresponding restrictions from Proposition 1

$$
\begin{aligned}
\alpha_{0} & \sim U(0, d) \\
\alpha_{1} & \sim U\left(0,2 e^{\gamma}\right) \\
\beta & \sim U(0,1)
\end{aligned}
$$

where $d$ is any positive value.

The corresponding joint posterior distribution is the product of the previous prior distributions and the $k$ th power of the pseudo-likelihood function (see e.g. Francq and Zakoian (2010)) 


$$
\begin{aligned}
\pi^{(k)}\left(\alpha_{0}, \alpha_{1}, \beta \mid \mathbf{y}\right) & \propto\left[L\left(\alpha_{0}, \alpha_{1}, \beta \mid \mathbf{y}\right)\right]^{k} \pi\left(\alpha_{0}\right) \pi\left(\alpha_{1}\right) \pi(\beta) \\
& \propto \prod_{i=1}^{N}\left(2 \pi \sigma_{i}^{2}\right)^{-\frac{k}{2}} \exp \left\{-k \frac{y_{i}^{2}}{2 \sigma_{i}^{2}}\right\} I_{(0, d)}\left(\alpha_{0}\right) I_{\left(0,2 e^{\gamma}\right)}\left(\alpha_{1}\right) I_{(0,1)}(\beta) .
\end{aligned}
$$

The conditional posterior distribution of the parameters are

$$
\begin{aligned}
\pi^{(k)}\left(\alpha_{0} \mid \alpha_{1}, \beta, \mathbf{y}\right) & \propto\left(\prod_{i=1}^{N} \sigma_{i}^{2}\right)^{-\frac{k}{2}} \exp \left\{-k \sum_{i=1}^{N} \frac{y_{i}^{2}}{2 \sigma_{i}^{2}}\right\} I_{(0, d)}\left(\alpha_{0}\right) \\
\pi^{(k)}\left(\alpha_{1} \mid \alpha_{0}, \beta, \mathbf{y}\right) & \propto\left(\prod_{i=1}^{N} \sigma_{i}^{2}\right)^{-\frac{k}{2}} \exp \left\{-k \sum_{i=1}^{N} \frac{y_{i}^{2}}{2 \sigma_{i}^{2}}\right\} I_{\left(0,2 e^{\gamma}\right)}\left(\alpha_{1}\right) \\
\pi^{(k)}\left(\beta \mid \alpha_{0}, \alpha_{1}, \mathbf{y}\right) & \propto\left(\prod_{i=1}^{N} \sigma_{i}^{2}\right)^{-\frac{k}{2}} \exp \left\{-k \sum_{i=1}^{N} \frac{y_{i}^{2}}{2 \sigma_{i}^{2}}\right\} I_{(0,1)}(\beta)
\end{aligned}
$$

The data cloning algorithm starts with an initial solution $\alpha_{0}^{(0)}, \alpha_{1}^{(0)}, \beta^{(0)}$, and generates values for $\alpha_{0}^{(m)}, \alpha_{1}^{(m)}, \beta^{(m)}$ from the previous conditional posterior distributions of the parameters in each iteration $m$.

The algorithm can be summarized in the next steps

Step 1 Set initial values $\alpha_{0}^{(0)}, \alpha_{1}^{(0)}, \beta^{(0)}$ at $m=0$.

Step 2: Generate $\alpha_{0}^{(m+1)}$ from

$$
\alpha_{0}^{(m+1)} \sim \pi^{(k)}\left(\alpha_{0} \mid \alpha_{1}^{(m)}, \beta^{(m)}, \mathbf{y}\right)
$$

Step 3: Generate $\alpha_{1}^{(m+1)}$ from

$$
\alpha_{1}^{(m+1)} \sim \pi^{(k)}\left(\alpha_{1} \mid \alpha_{0}^{(m+1)}, \beta^{(m)}, \mathbf{y}\right)
$$

Step 4: Generate $\beta^{(m+1)}$ from

$$
\beta^{(m+1)} \sim \pi^{(k)}\left(\beta \mid \alpha_{0}^{(m+1)}, \alpha_{1}^{(m+1)}, \mathbf{y}\right)
$$

Step 5: Set $m=m+1$. Go to Step 2 .

The data cloning algorithm has been programmed by using the package dclone (Sólymos (2010)) from the $\mathrm{R}$ project ( $\mathrm{R}$ Core Team (2012)). In order to check the performance of this algorithm in GARCH models, we have simulated data series from a known GARCH(1,1) model and we have compared the obtained results with the real ones.

We simulate, as an example, 1000 observations from a $\operatorname{GARCH}(1,1)$ model with parameters $\alpha_{0}=3, \alpha_{1}=0.7$ and $\beta=0.1$, by means of the R library TSA (Chan and Ripley (2012)).

We check what the optimal number of clones is, regarding some statistics computed in the package dclone (Sólymos (2010)), such as the maximum eigenvalue of the posterior variance, the minimum squared error and the squared error (see Lele et al. (2010) and Brooks and Gelman (1998)). There are not relevant improvements in their values when the number of clones is larger than 10 , therefore we use this number of clones to analyze the data.

Table 1 shows the real values of $\alpha_{0}, \alpha_{1}$ and $\beta$ from which data has been simulated, the parameters estimation, the standard errors and the $95 \%$ frequentist confidence intervals.

These results show that all parameters are quite close to their real values. Moreover, the standard errors are low and the $95 \%$ confidence intervals include all the real values. Similar results can be found with any other set of simulated parameters.

We compare now the data cloning method with a Bayesian MCMC algorithm using the same simulated data. The MCMC algorithm has been programmed using Jags (Plummer et al. (2003)) by means of the package runjags (Denwood (2011)) from the R project ( $\mathrm{R}$ Core Team (2012)). One advantage of using Jags is that it constructs the full conditional distributions and it carries 


\begin{tabular}{|c|c|c|c|c|}
\hline Parameter & Real Value & Estimator & S.D. & $95 \%$ Confidence Interval \\
\hline$\alpha_{0}$ & 3 & 3.229 & 0.333 & $(2.589,3.869)$ \\
\hline$\alpha_{1}$ & 0.7 & 0.744 & 0.075 & $(0.597,0.891)$ \\
\hline$\beta$ & 0.1 & 0.088 & 0.031 & $(0.027,0.148)$ \\
\hline
\end{tabular}

Table 1: Estimation for GARCH parameters using the data cloning method

out the Gibbs sampling from the model specifications. All codes are available from the authors, upon request.

In table 2 it is shown the posterior means, standard errors and the credible intervals for the GARCH model parameters when the prior distributions are the same as in the data cloning method (see, e.g., Nakatsuma (1998)).

\begin{tabular}{|c|c|c|c|c|}
\hline Parameter & Real Value & Posterior mean & S.D. & HPD 0.95 \\
\hline$\alpha_{0}$ & 3 & 3.226 & 0.328 & $(2.612,3.898)$ \\
\hline$\alpha_{1}$ & 0.7 & 0.743 & 0.074 & $(0.606,0.896)$ \\
\hline$\beta$ & 0.1 & 0.094 & 0.031 & $(0.042,0.163)$ \\
\hline
\end{tabular}

Table 2: Estimation for GARCH parameters using MCMC

Tables 1 and 2 show similar accurate results, although interpretations of them must be done in terms of frequentist or Bayesian point of views.

\subsection{Data cloning estimation of COGARCH models}

In this section we estimate the COGARCH model parameters under the data cloning methodology. As in section 4.1, at first it is necessary to consider the corresponding conditional posterior distributions of the parameters.

We consider the following vaguely informative prior distributions

$$
\begin{aligned}
& \beta \sim U(0, a) \\
& \varphi \sim U(0, c) \\
& \eta \sim U(\varphi, b)
\end{aligned}
$$

where $a, c$ and $b$ are positive real values and $b>c$.

The corresponding joint posterior distribution is the product of the previous prior distributions and the $k$ th power of the pseudo-likelihood function (see 7 ) given by

$$
\begin{aligned}
\pi^{(k)}(\beta, \varphi, \eta \mid \mathbf{y}) & \propto[L(\beta, \varphi, \eta \mid \mathbf{y})]^{k} \pi(\beta) \pi(\varphi) \pi(\eta) \\
& \propto\left(\prod_{i=1}^{N} \rho_{i}^{2}\right)^{-k / 2} \exp \left\{-k \sum_{i=1}^{N} \frac{y_{i}^{2}}{2 \rho_{i}^{2}}\right\} I_{(0, a)}(\beta) I_{(\varphi, b)}(\eta) I_{(0, c)}(\varphi) .
\end{aligned}
$$

Then, the conditional posterior distribution of the parameters are

$$
\begin{aligned}
& \pi^{(k)}(\beta \mid \varphi, \eta, \mathbf{y}) \propto\left(\prod_{i=1}^{N} \rho_{i}^{2}\right)^{-k / 2} \exp \left\{-k \sum_{i=1}^{N} \frac{y_{i}^{2}}{2 \rho_{i}^{2}}\right\} I_{(0, a)}(\beta) \\
& \pi^{(k)}(\varphi \mid \beta, \eta, \mathbf{y}) \propto\left(\prod_{i=1}^{N} \rho_{i}^{2}\right)^{-k / 2} \exp \left\{-k \sum_{i=1}^{N} \frac{y_{i}^{2}}{2 \rho_{i}^{2}}\right\} I_{(0, c)}(\varphi) \\
& \pi^{(k)}(\eta \mid \beta, \varphi, \mathbf{y}) \propto\left(\prod_{i=1}^{N} \rho_{i}^{2}\right)^{-k / 2} \exp \left\{-k \sum_{i=1}^{N} \frac{y_{i}^{2}}{2 \rho_{i}^{2}}\right\} I_{(\varphi, b)}(\eta)
\end{aligned}
$$


The data cloning algorithm generates values for $\beta^{(m)}, \varphi^{(m)}, \eta^{(m)}$ from the previous conditional posterior distributions of the parameters in each iteration $m$ from initial values $\beta^{(0)}, \varphi^{(0)}, \eta^{(0)}$.

The algorithm can be summarized in the next steps

Step 1 Set initial values $\beta^{(0)}, \varphi^{(0)}, \eta^{(0)}$ at $m=0$.

Step 2: Generate $\beta^{(m+1)}$ from

$$
\beta^{(m+1)} \propto\left(\prod_{i=1}^{N} \rho_{i}^{2}\right)^{-k / 2} \exp \left\{-k \sum_{i=1}^{N} \frac{y_{i}^{2}}{2 \rho_{i}^{2}}\right\} I_{(0, a)}(\beta)
$$

where

$$
\begin{aligned}
\rho_{i}^{2} & =\left(\sigma_{i-1}^{2}-\frac{\beta^{(m)}}{\eta^{(m)}-\varphi^{(m)}}\right)\left(\frac{\exp \left\{\left(\eta^{(m)}-\varphi^{(m)}\right) \Delta t_{i}\right\}-1}{\eta^{(m)}-\varphi^{(m)}}\right)+\frac{\beta^{(m)} \Delta t_{i}}{\eta^{(m)}-\varphi^{(m)}} \\
\sigma_{i-1}^{2} & =\beta^{(m)} \Delta t_{i-1}+\exp \left\{-\eta^{(m)} \Delta t_{i-1}\right\} \sigma_{i-2}^{2}+\varphi^{(m)} \exp \left\{-\eta^{(m)} \Delta t_{i-1}\right\} y_{i-1}^{2} \\
\sigma_{0}^{2} & =\frac{\beta^{(m)}}{\eta^{(m)}-\varphi^{(m)}}
\end{aligned}
$$

for $i=1, \ldots, N$.

Step 3: Generate $\varphi^{(m+1)}$ from

$$
\varphi^{(m+1)} \propto\left(\prod_{i=1}^{N} \rho_{i}^{2}\right)^{-k / 2} \exp \left\{-k \sum_{i=1}^{N} \frac{y_{i}^{2}}{2 \rho_{i}^{2}}\right\} I_{(0, c)}(\varphi)
$$

where

$$
\begin{aligned}
\rho_{i}^{2} & =\left(\sigma_{i-1}^{2}-\frac{\beta^{(m+1)}}{\eta^{(m)}-\varphi^{(m)}}\right)\left(\frac{\exp \left\{\left(\eta^{(m)}-\varphi^{(m)}\right) \Delta t_{i}\right\}-1}{\eta^{(m)}-\varphi^{(m)}}\right)+\frac{\beta^{(m+1)} \Delta t_{i}}{\eta^{(m)}-\varphi^{(m)}} \\
\sigma_{i-1}^{2} & =\beta^{(m+1)} \Delta t_{i-1}+\exp \left\{-\eta^{(m)} \Delta t_{i-1}\right\} \sigma_{i-2}^{2}+\varphi^{(m)} \exp \left\{-\eta^{(m)} \Delta t_{i-1}\right\} y_{i-1}^{2} \\
\sigma_{0}^{2} & =\frac{\beta^{(m+1)}}{\eta^{(m)}-\varphi^{(m)}}
\end{aligned}
$$

for $i=1, \ldots, N$.

Step 4: Generate $\eta^{(m+1)}$ from

$$
\eta^{(m+1)} \propto\left(\prod_{i=1}^{N} \rho_{i}^{2}\right)^{-k / 2} \exp \left\{-k \sum_{i=1}^{N} \frac{y_{i}^{2}}{2 \rho_{i}^{2}}\right\} I_{\left(\varphi^{(m+1)}, b\right)}(\eta)
$$

where

$$
\begin{aligned}
\rho_{i}^{2} & =\left(\sigma_{i-1}^{2}-\frac{\beta^{(m+1)}}{\eta^{(m)}-\varphi^{(m+1)}}\right)\left(\frac{\exp \left\{\left(\eta^{(m)}-\varphi^{(m+1)}\right) \Delta t_{i}\right\}-1}{\eta^{(m)}-\varphi^{(m+1)}}\right)+\frac{\beta^{(m+1)} \Delta t_{i}}{\eta^{(m)}-\varphi^{(m+1)}} \\
\sigma_{i-1}^{2} & =\beta^{(m+1)} \Delta t_{i-1}+\exp \left\{-\eta^{(m)} \Delta t_{i-1}\right\} \sigma_{i-2}^{2}+\varphi^{(m+1)} \exp \left\{-\eta^{(m)} \Delta t_{i-1}\right\} y_{i-1}^{2} \\
\sigma_{0}^{2} & =\frac{\beta^{(m+1)}}{\eta^{(m)}-\varphi^{(m+1)}}
\end{aligned}
$$

for $i=1, \ldots, N$.

Step 5: Set $m=m+1$. Go to Step 2 .

In order to check the performance of the data cloning method, we have simulated data series from a known COGARCH model and we have compared the parameters estimations with the real ones from which data has been generated.

We simulate, as an example, 180 observations from a COGARCH with parameters $\beta=0.35$, $\eta=0.25$ and $\phi=0.02$ following the same procedure as in Maller et al. (2008).

As in section 4.1, we check what the optimal number of clones is in term of the statistics computed in the package dclone (Sólymos (2010)). In this case there are not relevant improvements 
in the values when the number of clones is larger than 20, therefore we use this number of clones to analyze the data.

Table 3 shows the real values of $\beta, \eta$ and $\phi$, the parameters estimations, the standard errors and the $95 \%$ frequentist confidence intervals.

\begin{tabular}{|c|c|c|c|c|}
\hline Parameter & Real Value & Estimators & S.D. & $95 \%$ Confidence Interval \\
\hline$\beta$ & 0.25 & 0.268 & 0.211 & $(0,0.682)$ \\
\hline$\eta$ & 0.35 & 0.188 & 0.146 & $(0,0.474)$ \\
\hline$\phi$ & 0.02 & 0.045 & 0.021 & $(0.003,0.086)$ \\
\hline
\end{tabular}

Table 3: Estimation for COGARCH parameters using data cloning

These results show that all parameters are quite close to their real values, the standard errors are low and the $95 \%$ confidence intervals include all the real values. Similar results can be found with other sets of simulated parameters.

In Table 4 it is shown the posterior means, standard errors and the credible intervals for the parameters of the $\mathrm{COGARCH}$ model, using a Bayesian MCMC approach when the prior distributions are the same used in the data cloning algorithm.

\begin{tabular}{|c|c|c|c|c|}
\hline Parameter & Real Value & Estimated Value & S.D. & HPD 0.95 \\
\hline$\beta$ & 0.25 & 0.427 & 0.158 & $(0.138 ; 0.747)$ \\
\hline$\eta$ & 0.35 & 0.301 & 0.132 & $(0.092 ; 0.600)$ \\
\hline$\phi$ & 0.02 & 0.029 & 0.014 & $(0.002 ; 0.049)$ \\
\hline
\end{tabular}

Table 4: Estimation for COGARCH parameters using MCMC

Both tables 3 and 4, show similar accurate results, although interpretations of parameters are different.

\section{Data cloning estimation for a NASDAQ-100 stock index data set}

GARCH and COGARCH models have been proposed to deal with volatilities in real financial series, assuming stationarity. Therefore, in this section we apply GARCH(1,1) and COGARH models to analyze the behavior of Nasdaq-100 index series. In order to obtain estimators of the parameters, we apply both, data cloning and full Bayesian methodologies.

The data are obtained from the website finance.yahoo.com and we consider weekly returns of Nasdaq-100 index from January 2000 to November 2012.

We first test the presence of autoregressive conditional heteroskedasticity effects in the series, by means of the $A R C H-L M$ test (see Godfrey (1978) and Breusch (1978)). Table 5 shows the LM statistic and the corresponding p-values computed on the Nasdaq-100 returns. It can be concluded that this series presents autoregressive conditional heteroskedasticity effects.

\begin{tabular}{|c|c|c|}
\hline lags & $L M$ & p-value \\
\hline 1 & 20.2287 & $6.871 \mathrm{e}-06$ \\
5 & 40.6069 & $1.126 \mathrm{e}-07$ \\
10 & 82.096 & $1.947 \mathrm{e}-13$ \\
15 & 179.5892 & $2.2 \mathrm{e}-16$ \\
20 & 212.5089 & $2.2 \mathrm{e}-16$ \\
\hline
\end{tabular}

Table 5: Lagrange Multipliplier test for Nasdaq-100 returns 
Then we check which are the most appropriate model by using the Akaike information criterion (AIC) (Akaike (1974)) and the Bayesian information criterion (BIC) (Schwarz (1978)). Their respective values are shown in table 6 .

\begin{tabular}{|c|c|c|}
\hline Model & AIC & BIC \\
\hline ARCH $(1)$ & 2381.906 & -2372.885 \\
ARCH $(2)$ & -2538.131 & -2524.601 \\
GARCH $(1,1)$ & -2623.244 & -2609.713 \\
GARCH $(1,2)$ & -2614.217 & -2596.176 \\
GARCH $(2,1)$ & -2606.293 & -2588.252 \\
GARCH $(2,2)$ & -2611.563 & -2589.012 \\
\hline
\end{tabular}

Table 6: AIC and BIC criteria for several GARCH models

Minimum values for AIC and BIC criteria are found just for a $\mathrm{GARCH}(1,1)$ model, therefore it will be used for modeling the behavior of the weekly Nasdaq-100 returns series.

We use the data cloning approach, as in section 4.1, to estimate the parameters, their standard errors and the approximate maximum likelihood intervals. We check what the optimal number of clones is (see (Sólymos (2010)) and we obtain that there are not relevant improvements when the number of clones is larger than 40, therefore we use this number of clones to analyze the data.

Using 40 clones, the results are shown in Table 7.

\begin{tabular}{|c|c|c|c|}
\hline Parameter & Estimated Value & S.D. & Confidence Interval 95\% \\
\hline$\alpha_{0}$ & $7.82 e-05$ & $2.97 e-06$ & $(4.13 e-05 ; 1.15 e-04)$ \\
\hline$\alpha_{1}$ & 0.196 & $5.01 e-03$ & $(0.134 ; 0.258)$ \\
\hline$\beta$ & 0.741 & $4.60 e-03$ & $(0.684 ; 0.798)$ \\
\hline
\end{tabular}

Table 7: Clone estimations for $\operatorname{GARCH}(1,1)$ parameters to model Nasdaq weekly returns

Results obtained with data cloning can be compared with those obtained by a standard MCMC Bayesian approach (see e.g. Nakatsuma (1998)). Table 8 shows posterior means, standard errors and the credible intervals for the $\operatorname{GARCH}(1,1)$ parameters under a full Bayesian approach.

\begin{tabular}{|c|c|c|c|}
\hline Parameter & Estimated Value & S.D. & HPD 0.95 \\
\hline$\alpha_{0}$ & $8.31 e-05$ & $1.94 e-05$ & $(4.90 e-05 ; 1.25 e-04)$ \\
\hline$\alpha_{1}$ & 0.2054 & 0.0324 & $(0.147 ; 0.2748)$ \\
\hline$\beta$ & 0.7324 & 0.0285 & $(0.675 ; 0.7863)$ \\
\hline
\end{tabular}

Table 8: MCMC estimations for $\operatorname{GARCH}(1,1)$ parameters to model Nasdaq weekly returns

Both, data cloning and Bayesian methodologies, show similar results (see table 7 and table 8).

We now analyze the Nasdaq-100 daily returns from January 2008 to December 2012. As COGARCH model enable to analyze irregularly spaced time series, we will use it to take into account holiday periods and weekends of the daily Nasdaq-100 series.

As in section 4.2, we use the data cloning approach to estimate the parameters of the COCARCH model, their standard errors and the approximate frequentist intervals. We also consider a MCMC Bayesian approach in order to obtain the posterior distributions and the credible intervals of the parameters. Results are shown in table 9 and table 10, respectively. 


\begin{tabular}{|c|c|c|c|}
\hline Parameter & Estimated Value & S.D. & Confidence Interval 95\% \\
\hline$\beta$ & 9.041 & 0.289 & $(5.457 ; 12.626)$ \\
\hline$\eta$ & 0.055 & 0.00042 & $(0.050 ; 0.061)$ \\
\hline$\phi$ & 0.05 & 0.00015 & $(0.048 ; 0.052)$ \\
\hline
\end{tabular}

Table 9: Clone estimations of COGARCH parameters to model Nasdaq daily returns

\begin{tabular}{|c|c|c|c|}
\hline Parameter & Estimated Value & S.D. & HPD 0.95 \\
\hline$\beta$ & 9.661 & 2.113 & $(6.140 ; 14.414)$ \\
\hline$\eta$ & 0.054 & 0.0043 & $(0.045 ; 0.063)$ \\
\hline$\phi$ & 0.047 & 0.0028 & $(0.039 ; 0.050)$ \\
\hline
\end{tabular}

Table 10: MCMC estimations of COGARCH parameters to model Nasdaq daily returns

Tables 9 and 10 show similar results in the estimations and the standard errors seem to be lower for the data cloning algorithm.

Parameter $\eta$ measures the speed of the decline of a volatility burst. For this dataset the value is not very high, namely, when a volatility burst appears due to the arrival of new information to markets, its influence in volatility declines with a moderate speed.

Parameter $\phi$ measures the magnitude of the volatility jumps and it may be considered as a measure of how information affects to volatility and how fast market assumes new events. In Nasdaq-100 series the estimation of $\phi$ is quite large in relation to the values it may reach in order to maintain stationarity. A possible interpretation is that volatility bursts are quite big and daily index moves largely up or down regarding the appearance of relevant information in market.

Finally, parameter $\beta$ represents the level of volatility and regarding the Nasdaq-100 values, the estimated value is not very large.

\section{Final conclusions}

GARCH modeling is a very popular methodology that takes into account the most important stylized facts that financial series present and it is applied in equally spaced data. But there are periods of time with holidays and weekends that may affect the behavior of series, and it is convenient to introduce unequally spaced or continuous time periods. In this sense, COGARCH models may be a good alternative to tackle with this kind of situations.

In this work, we use COGARCH models based in a Lévy process which include volatility and model the process jumps (see Klüppelberg et al. (2004)). We propose to use a MCMC Bayesian algorithm and the data cloning methodology.

The main results are

(i) Either the posterior means estimated by a MCMC Bayesian method or the data cloning estimators can be used for estimating the parameters of GARCH and COGARCH models as they show a good behavior in the simulation study.

(ii) We have used GARCH and COGARCH models to analyze the behavior of the Nasdaq-100 index series, by using both data cloning and a MCMC Bayesian methodologies. Results are easily interpreted from a practical point of view.

\section{References}

Akaike, H. (1974). A new look at the statistical model identification. Automatic Control, IEEE Transactions on 19(6), 716-723.

Ardia, D. (2008). Financial Risk Management with Bayesian Estimation of GARCH Models: Theory and Applications, Volume 612. Springer. 
Bollerslev, T. (1986). Generalized autoregressive conditional heteroskedasticity. Journal of econometrics 31(3), 307-327.

Bollerslev, T. (2008). Glossary to ARCH (GARCH). Russell The Journal Of The Bertrand Russell Archives 49.

Breusch, T. (1978). Testing for autocorrelation in dynamic linear models*. Australian Economic Papers 17(31), 334-355.

Brooks, S. and A. Gelman (1998). General methods for monitoring convergence of iterative simulations. Journal of computational and graphical statistics 7(4), 434-455.

Chan, K.-S. and B. Ripley (2012). TSA: Time Series Analysis. R package version 1.01.

Corradi, V. (2000). Reconsidering the continuous time limit of the garch $(1,1)$ process. Journal of Econometrics 96(1), 145-153.

Denwood, M. J. (2011). runjags: Run Bayesian MCMC Models in the BUGS syntax from Within $R$. R package version $0.9 .9-2$.

Engle, R. (1982). Autoregressive conditional heteroscedasticity with estimates of the variance of united kingdom inflation. Econometrica 50(4), 987-1008.

Francq, C. and J. Zakoian (2010). GARCH models: structure, statistical inference and financial applications. Wiley.

Godfrey, L. (1978). Testing against general autoregressive and moving average error models when the regressors include lagged dependent variables. Econometrica: Journal of the Econometric Society, 1293-1301.

Haug, S., C. Klüppelberg, A. Lindner, and M. Zapp (2007). Method of moment estimation in the cogarch $(1,1)$ model. The Econometrics Journal 10(2), 320-341.

Kallsen, J. and M. Taqqu (1998). Option pricing in arch-type models. Mathematical Finance 8(1), $13-26$.

Kazmerchuk, Y., A. Swishchuk, and J. Wu (2005). A continuous-time garch model for stochastic volatility with delay. Canadian Applied Mathematics Quarterly 13(2), 123-149.

Klüppelberg, C., A. Lindner, and R. Maller (2004). A continuous-time garch process driven by a lévy process: Stationarity and second-order behaviour. Journal of Applied Probability 41(3), $601-622$.

Lele, S., B. Dennis, and F. Lutscher (2007). Data cloning: easy maximum likelihood estimation for complex ecological models using bayesian markov chain monte carlo methods. Ecology Letters 10(7), 551-563.

Lele, S., K. Nadeem, and B. Schmuland (2010). Estimability and likelihood inference for generalized linear mixed models using data cloning. Journal of the American Statistical Association 105(492), 1617-1625.

Lorenz, R. (2006). Weak approximation of stochastic delay differential equations with bounded memory by discrete time series. Ph. D. thesis, Ph. D. thesis, Humbold University of Berlin, Berlin, Germany.

Maller, R., G. Müller, and A. Szimayer (2008). Garch modelling in continuous time for irregularly spaced time series data. Bernoulli 14 (2), 519-542.

Mandelbrot, B. (1963). The variation of certain speculative prices. The journal of business 36(4), 394-419.

Müller, G. (2010). Mcmc estimation of the cogarch $(1,1)$ model. Journal of Financial Econometrics $8(4), 481-510$. 
Nakatsuma, T. (1998). A markov-chain sampling algorithm for garch models. Studies in Nonlinear Dynamics 8 Econometrics $3(2)$.

Nelson, D. (1990). Arch models as diffusion approximations. Journal of Econometrics 45(1-2), $7-38$.

Plummer, M. et al. (2003). Jags: A program for analysis of bayesian graphical models using gibbs sampling. URL http://citeseer. ist. psu. edu/plummer03jags. html.

R Core Team (2012). R: A Language and Environment for Statistical Computing. Vienna, Austria: R Foundation for Statistical Computing. ISBN 3-900051-07-0.

Schwarz, G. (1978). Estimating the dimension of a model. The annals of statistics 6(2), 461-464.

Sólymos, P. (2010). dclone: Data cloning in R. The R Journal 2(2), 29-37.

Wang, Y. (2002). Asymptotic nonequivalence of garch models and diffusions. The Annals of Statistics 30(3), 754-783. 